\title{
Cabildo, políticas económicas y mercados en el Río de la Plata y otros puntos de Hispanoamérica colonial (siglos XVI-XVIII)
}

\author{
Mauro Luis Pelozatto Reilly ${ }^{1}$
}

\section{Fundamentación}

Desde hace un tiempo a esta parte, he venido encarando distintas investigaciones concentradas en el accionar del cabildo hispano-colonial, centrándome principalmente en las políticas económicas dispuestas sobre las principales actividades y sus mercados, según el contexto geográfico y temporal que hayan sido considerados, siempre partiendo de la base de que se trató de una institución con múltiples atribuciones, y de que sus intervenciones influyeron no solamente sobre la economía, sino también sobre los intereses de los distintos sectores y actores sociales vinculados a la misma y sus variantes.

El objetivo principal del presente trabajo consiste en hacer un repaso analítico de una serie de ponencias que tuve la posibilidad de exponer en distintos congresos y jornadas de carácter académico durante el último año. Estas intervenciones fueron resultado de trabajos elaborados en torno al eje temático definido por la relación entre el ayuntamiento y sus medidas sobre cuestiones productivas, comerciales e impositivas, las cuales tuvieron un desarrollo particular, según el caso (por ejemplo, con un mayor énfasis en la minería de la plata y sus problemáticas en la Ciudad de Zacatecas, entre fines del siglo XVI y comienzos del siguiente, o concentradas mayormente en la ganadería y sus alternativas mercantiles en Santa Fe de la Vera Cruz durante la primera mitad del siglo XVIII, como para mencionar algunas diferencias iniciales), variando también las materias abordadas y las consecuencias socioeconómicas.

Se tuvieron en cuenta los variados puntos desarrollados en cada trabajo, las similitudes y diferencias entre los mismos, los diferentes objetos de estudio planteados, los problemas que pueden llegar a enumerarse, la fundamentación de los mismos, los objetivos de investigación, las hipótesis planteadas,

Argentino. Profesor en Historia, Universidad de Morón (UM), Magíster en Ciencias Sociales con mención en Historia Social, Universidad Nacional de Luján (UNLu). Académico, Universidad Nacional de La Matanza (UNLaM), Argentina. E-mail: maurolpr@hotmail.com.ar 
así como también otras cuestiones de índole teórica (conceptos, hipótesis previas e ideas de otros especialistas) y metodológica (fuentes, métodos, tipos de datos, perspectivas de análisis, etc.), con la idea de finalizar el escrito planteando algunas aproximaciones parciales, interrogantes por responder y posibles líneas de investigación pensadas a futuro.

\section{Diferentes investigaciones sobre una misma materia: el abasto de carne local en Hispanoamérica colonial (Ecuador y Litoral Rioplatense)}

Uno de los temas que me ha permitido sostener el planteo de que las tratativas capitulares sobre un mismo asunto o conjunto de problemáticas de índole económica pueden variar según el momento y el lugar ha sido la relación entre las políticas públicas municipales y el abasto de carne del vecindario.

Para argumentar dicha formulación, me propongo en este apartado repasar algunos ítems extraídos de mis investigaciones sobre el mencionado eje temático, las cuales se concentraron principalmente en jurisdicciones del actual territorio ecuatoriano durante la segunda mitad del siglo XVI (Quito y Guayaquil), y de la Provincia del Río de la Plata desde comienzos del siglo XVIII (Buenos Aires y Santa Fe). Dentro del repaso, se tienen en cuenta tanto similitudes como diferencias en cuanto a las regulaciones concejiles y las características productivas y comerciales de cada espacio. También es preciso remarcar algunos puntos en relación a lo metodológico, como las fuentes y cómo encaré el análisis de las mismas para la elaboración de distintos tipos de datos pertinentes.

Respecto al primer conjunto de ciudades, el año pasado realicé una exposición en unas jornadas de Historia Argentina y Latinoamericana, en torno a las cuales creo conveniente señalar algunos puntos del planteo inicial, del desarrollo, las fuentes, la metodología, el marco conceptual y las aproximaciones finales.

En lo tocante al objeto de estudio, el mismo consiste en hacer una enumeración y caracterización de las principales atribuciones del concejo en relación a las actividades productivas agropecuarias más destacadas y sus mercados, con base en el análisis de las Ordenanzas del Cabildo de Quito (1568) y las del de Guayaquil (1590), es decir, concentrándome en supuestos y no en situaciones concretas. Esta última aclaración resulta de utilidad, ya que para mis próximas indagaciones me propongo hacer un contraste entre lo señalado por el Derecho Indiano y la práctica. Es por eso que creo que esta investigación consiste en una aproximación inicial y parcial (Pelozatto Reilly, 2017d: 1). 
Como punto diferencial de aquella ponencia, me gustaría mencionar la importancia que di a los productos agrícolas (según el lugar), y al señalamiento y la explicación de las similitudes y diferencias entre el accionar de ambos ayuntamientos sobre el bastimento cárnico (Pelozatto Reilly, 2017d: 2-3). Desde el punto de vista teórico y conceptual, el trabajo cuenta con un estado de la cuestión bastante completo, en el que se destacan términos aplicables al estudio de las fuentes seleccionadas, tales como el de "funciones económicas del cabildo" (dentro de las cuales estaban el nombramiento de un obligado de abastecer los corrales urbanos, de regular las obligaciones del mismo, negociar los precios, las cantidades y la calidad, controlar la venta de la carne y del ganado, etc.) (Guerrero Rincón, 1997: 81-82); el de la política proteccionista del Antiguo Régimen (ya que el cuerpo se encargaba de limitar el comercio, ajustar los valores monetarios, aplicar cargas fiscales, verificar la recaudación, controlar el matadero y las faenas, tomar medidas contra el comercio ilegal, entre otras cosas) (Moncada González, 2009: 469-488); y la idea de que el ayuntamiento seguía una lógica tanto humanista como mercantilista (Quiroz, 2011: 35-59), en el sentido de que debía garantizar que todos sus pobladores accedieran al consumo del alimento en cuestión, y al mismo tiempo debían quedar ganancias para los reseros y mercaderes (Pelozatto Reilly, 2017d: 3-6). En cuanto a las conclusiones, más allá de señalar la multiplicidad de materias abordadas por la sala capitular en cada caso, me encargué de marcar importantes diferencias entre ambos, sobre todo en lo que tiene que ver con los sistemas de aprovisionamiento de carne, la importancia cuantitativa de la ganadería en cada localidad, los distintos tipos de cultivos (que variaban, por lógicas razones), la designación de diversos funcionarios en relación al matadero y las carnicerías, etc.

Tomando otra región marcadamente distinta (Litoral Rioplatense), durante la primera mitad del siglo XVIII, y concentrándome más que nada en el análisis de documentos municipales, pude marcar puntos de coincidencia y de disimilitud entre el desarrollo de la ganadería y del mercado de la carne en Buenos Aires y Santa Fe. Sobre los primeros, se podría destacar el remate en pública almoneda del derecho de abasto de carne, que recaía sobre el mejor postor (mecanismo frecuente en ambos municipios), la obligación directa a los hacendados de acercar sus reses al mercado citadino (sobre todo en períodos de carestía o de acentuada especulación), la participación activa de los integrantes del cuerpo capitular en cuestiones de impuestos, precios, controles de calidad, cantidades, pesos y medidas, marcas y señales, permisión o prohibición de matanzas, etc. Como principales diferencias, aparecen un mayor predominio del sistema de abastecimiento de corrales en Santa Fe, y una evolución distinta del ganado vacuno disponible en cada lugar: mientras en Buenos Aires las vaquerías de caza tuvieron peso hasta por lo menos 1723 (año de reconocimiento de la última acción sobre animales cimarrones), en Santa Fe ya desde comienzos de siglo hubo limitaciones y hasta cierres de 
este tipo de actividades por falta de recursos. Asimismo, tanto la demanda de alimentos como las cantidades de cabezas sacrificadas para el abasto fueron mucho más abultadas en el caso porteño que en el santafesino. Por otra parte, también pude ocuparme de conflictos puntuales por el aprovechamiento de las haciendas, como el caracterizado por las discusiones en torno a la concordia establecida entre el Cabildo de Buenos Aires, su par de la ciudad restante y los representantes de la Compañía de Jesús, que terminó, luego de varias idas y venidas, en una redistribución desigual de los vacunos salvajes de los campos entrerrianos y orientales, viéndose claramente beneficiados los bonaerenses y los jesuitas por sobre los intereses de Santa Fe (Pelozatto Reilly, 2015; 2017a).

Para agregar algo distinto al otro avance descrito, sobre todo teniendo en cuenta la realidad de los productores y abastecedores de Buenos Aires, me encuentro acercándome a cuestiones como el perfil productivo de los estancieros de la zona, los mercados a los cuales se encontraban ligados, la composición interna de los establecimientos productivos, las distintas situaciones ocupacionales, laborales y de vida de los trabajadores rurales, la importancia de los pequeños y medianos productores rurales, la relación entre los estancieros y el cabildo, la representación de sus intereses dentro de aquel, la participación de toda una variedad de actores económicos entre la producción en el campo y el consumo en la ciudad, etc. Todo ello gracias a la triangulación de una mayor cantidad y diversidad de fuentes históricas, más allá de las actas capitulares, como los documentos compilados en el archivo de la mencionada institución, las sucesiones, testamentos e inventarios de las propiedades de los vecinos vinculados a la cría y comercialización de animales, y los padrones rurales (Pelozatto Reilly, 2016).

Como deuda, tengo el realizar estudios parecidos tomando los productores rurales santafesinos y su vinculación tanto con el ayuntamiento como con las distintas posibilidades comerciales en torno a la ganadería y la agricultura.

\section{Un ejemplo de historia local y regional comparada en torno a varias cuestiones sobre ganadería: Santa Fe y San Luis (1700-1750)}

Indudablemente, el objeto de estudio planteado al principio es atravesado por la perspectiva que los propone la historia regional comparada, ya que los principales objetivos de la investigación tomada para este apartado consisten en analizar, desde un punto de vista comparativo, varios aspectos en distintos espacios locales durante el mismo periodo de tiempo (1700-1750): el desarrollo de las prácticas productivas pecuarias, de la ganadería vacuna y sus alternativas mercantiles, el carácter de las políticas económicas municipales y el 
lugar ocupado por otras especies animales que representaban oportunidades económicas menores -desde la óptica de los productores, de los comerciantes y de los representantes del Estado colonial-, entre otros detalles influyentes, en las jurisdicciones de los cabildos de Santa Fe (Gobernación del Río de la Plata) y San Luis (Reino de Chile) (Pelozatto Reilly, 2017e: 1-2).

Luego de la fundamentación del tema, y de marcar como punto de partida las hipótesis de que durante el periodo estudiado tuvieron lugar en ambas regiones procesos de importantes cambios sobre la ganadería bovina en particular (como la extinción del ganado cimarrón y la consolidación de alternativas, y como las recogidas de animales alzados o la mayor preocupación por la reproducción del stock pecuario dentro de las estancias, transición bastante explicada para la campaña bonaerense) (Coni, 2011; Pelozatto Reilly, 2014a), se dio lugar a la exposición del desarrollo, el cual fue dividido en varias partes: a) la clasificación y explicación de las intromisiones capitulares; b) la caracterización del proceso de extinción de las reservas de vacunos salvajes y de los mecanismos llevados a la práctica por los cabildantes para contrarrestar sus efectos negativos en ambos sitios, y c) un intento de aproximación a los mercados que se le presentaban a los bienes ganaderos.

En lo que respecta a lo más estrictamente metodológico, hay varias cosas a tener presente. Primeramente, la utilización de la diversa información que nos brindan fuentes como las Actas del Cabildo de Santa Fe (donde aparecen las discusiones y resoluciones de las sesiones concejiles en torno a todas las problemáticas y asuntos de interés municipal, autos de gobernadores, reales provisiones emitidas por la Real Audiencia de La Plata, copias de cédulas firmadas por Su Majestad, cartas entre el ayuntamiento y distintos funcionarios, inventarios de las existencias de ganado dentro de la jurisdicción, peticiones de vecinos, licencias administradas por el municipio, registros de marcas y señales oficiales para los bienes de hacienda, etc.), y las Actas Capitulares de San Luis (en pocas palabras, documentos muy similares). Asimismo, los datos fueron elaborados y posteriormente expuestos de forma cualitativa (enumeración de ejemplos, descripciones y explicaciones sobre los mismos) y cuantitativa (elaboración y exposición de cifras, porcentajes y gráficos, etc.). También se tuvo en cuenta diferentes variables e indicadores, según el tema: por ejemplo, para cuantificar y describir los distintos mercados pecuarios, se consideraron las regulaciones capitulares sobre las recogidas de ganado (vacuno y equino, por separado), la administración de licencias, los controles sobre las reservas, la organización de las vaquerías de caza, el reconocimiento de acciones², el aprovisionamiento de las milicias, las medidas comerciales, el

Derechos de propiedad de los vecinos criadores sobre los animales cimarrones que pastaban libremente por la campaña. 
control de las estancias, la aplicación de impuestos a la cría y al comercio de ciertos animales (como las mulas, sobre las cuales pesaba el famoso cuartiIlo), las limitaciones sobre las faenas de sebo y grasa, la delimitación de los espacios productivos ${ }^{3}$, etc. (Pelozatto Reilly, 2017e: 3-4).

Por otro lado, para sostener la extinción de las vaquerías tradicionales, ${ }^{4}$ para ambas ciudades se realizó, entre otras cosas, un gráfico que muestra la evolución en las cantidades de resoluciones en torno a cuestiones de ganado vacuno cimarrón, extinto en Santa Fe desde por lo menos comienzos de la década de 1730, y en San Luis desde los primeros años de la centuria (pese a la breve aparición de planteles de ese tipo hacia 1750), apoyado con la mención y profundización de ejemplos concretos (Pelozatto Reilly, 2017e: 10). Simultáneamente, los datos muestran una creciente relevancia de las recogidas de alzados y de las decisiones del cuerpo sobre la cría en las unidades productivas (Pelozatto Reilly, 2015; 2016; 2017e: 11).

En lo que toca a las demás variantes de la ganadería, en el trabajo analizado me encargué de ejemplificar sobre las medidas más destacadas de la corporación local en relación a la ganadería mular (con un mayor peso de la imposición de gravámenes sobre los envíos hacia otras regiones desde Santa $\mathrm{Fe}$, y del control de las bestias que se introducían en los territorios agrícolas causando daños, como materia más interesante en San Luis), y a otras tramas de la actividad pastoril de menor peso, encontrándome con el destacable peso de la organización y la llevada a la práctica de las caballadas y potreadas ${ }^{5}$, tras haber realizado una comparación numérica con las que se practicaban sobre los bóvidos (Pelozatto Reilly, 2017e: 19).

Como conclusiones (Pelozatto Reilly, 2017e: 20), pude llegar a argumentar la idea de que el cabildo colonial se desempeñó en ambas partes como un organismo con múltiples atribuciones y como una corporación dentro de la cual

3 Espacios correspondientes, según los primeros repartos de tierras que tuvieron lugar desde las fundaciones de las ciudades, a las unidades productivas más especializadas en la agricultura (quintas, chacras, etc.), y las más orientadas a la cría y el mantenimiento de haciendas (estancias), o bien a una producción más diversificada (como las "estancias de cercanías").

4 Concepto de elaboración propia, elaborado para distinguir los distintos tipos de vaquerías: en este caso, se hace referencia a las expediciones de caza organizadas por el cabildo y los vecinos hacendados, las cuales eran practicadas sobre el ganado vacuno salvaje o cimarrón, con el principal fin de obtener cueros, piezas de sebo y grasa. Vale la pena aclarar que también aparecen denominadas como vaquerías las que prefiero mencionar como recogidas de ganado, las cuales se practicaban sobre los animales que se dispersaban por los campos, con el principal objetivo de reintegrarlos a las explotaciones rurales de sus dueños, o bien para conseguir reses para el abasto de carne local, entre otras cosas.

5 Dicho término (utilizado en los testimonios de la época) hace mención de las recogidas de equinos que, de manera similar que las que se hacían sobre vacunos (y muchas veces, ambas en simultáneo), eran encabezadas por los funcionarios correspondientes y por los interesados en los mencionados bienes de haciendas. 
se veían representados y enfrentados los intereses de las oligarquías locales (Fradkin y Garavaglia, 2009: 154); que en el caso santafesino hubo una mayor coexistencia entre las expediciones de caza y las recolecciones de bovinos dispersos; que en ambas jurisdicciones se presentaron diversas posibilidades lucrativas para los productos de la ganadería; la relevancia del abasto de carne local para los dos ayuntamientos en cuestión (que lo resolvieron a través de la práctica de varios sistemas $\left.{ }^{6}\right)$; que ambas Repúblicas funcionaron como abastecedoras de mulares para el norte minero dentro de lo que se conoce como espacio peruano ${ }^{7}$ (Assadourian, 1982: 270-273; Fradkin y Garavaglia, 2009: 65; Halperín Donghi, 2010: 43), y que tuvieron lugar otras variantes como las presentadas por los ovinos (obtención de carne y lana), los caballos y los bueyes (además de servir para la carga y el transporte, estos dos últimos cuadrúpedos sirven como indicadores de presencia agrícola en las estancias, o bien para medir la importancia de los mismos dentro de las explotaciones más específicamente agrarias (González Lebrero, 1993; Garavaglia, 1999).

\section{Dos estudios bien diferentes correspondientes a la misma época: Buenos Aires y Zacatecas entre fines del siglo XVI y comienzos del siglo XVII}

Para esta sección del artículo intento mostrar dos estudios de casos bien distintos, tomando como referencia el análisis de las políticas del cabildo colonial en dos puntos muy lejanos casi en la misma época, para sostener que las resoluciones del cuerpo variaban según los intereses económicos de cada lugar.

Por ejemplo, tras abordar las problemáticas y las mediaciones económicas y productivas del Cabildo de Buenos Aires entre 1580-1590, me encontré con que las mismas se concentraban en cuestiones como la concesión de mercedes de tierras y de repartimientos de indios, la conformación de las primeras unidades rurales (quintas, chacras, estancias, etc.), la guarda de los caballos ${ }^{8}$ y las vacas en los corrales de la ciudad, en controlar la delimitación

6 Entre esos sistemas estaban el estanco, la obligación directa a los productores y el "abastecimiento libre" de los corrales del mercado (predominantes en Santa Fe), y el prorrateo (reparto del número de cabezas a vender según la cantidad de vacunos domésticos que tuviera cada estanciero, más común en San Luis).

7 Entendido como el espacio económico que fue determinando la especialización local y regional de las zonas consideradas periféricas en torno a las demandas de alimentos, bienes de consumo, insumos y servicios, por parte de los principales centros políticos, productivos y comerciales (como Lima y varios puntos del Alto Perú).

8 Tanto en Buenos Aires como en Santa Fe, la designación de un guardacaballos (obligación que generalmente se remataba y que recaía sobre un vecino que estaba en condiciones económicas de hacerse cargo de las posibles pérdidas), fue una materia de interés para 
de los espacios productivos, en imponer multas para los criadores infractores, en representar los intereses de los labradores y de los pastores, y en ejercer controles sobre la propiedad efectiva de las cabezas de ganado (mediante la oficialización de las marcas y señales -yerros-, correspondientes a cada hacendado) (Pelozatto Reilly, 2017b).

En cambio, en Zacatecas, una ciudad principalmente minera, las medidas se concentraron casi exclusivamente en problemáticas mineras, dentro de las cuales se destacaron el nombramiento de funcionarios, el aprovisionamiento de la mano de obra y de insumos, los repartos de tierras y de indios, la regulación de los servicios y gastos, y la toma de medidas de seguridad (como las que se dieron ante la amenaza representada por los "indios infieles" sobre los reales de minas) (Pelozatto Reilly, 2017c: 1). Así, en la introducción me enfoqué, además de en reseñar distintos estudios locales y artículos elaborados desde varias perspectivas, en tomar algunas hipótesis previas para contrastar con lo encontrado en las Cartas de Cabildos Seculares, como la de entender a la minería (y particularmente a la argentífera) no solamente como actividad productiva principal, sino también como motor de las economías locales y regionales de las zonas periféricas (en este caso, las de Nueva España), en función de las necesidades de las áreas centrales (Pelozatto Reilly, 2017c: 2). Al mismo tiempo seleccioné otras consideraciones, como la mayoría representada por la mano de obra indígena (Gil Montero, 2012) -y de allí la relevancia de los repartimientos de indios encabezados por el cabildo-, la concentración de la producción platera en determinados puntos como lo fue Zacatecas (Commons, 1989), la importancia de los "polos de atracción" mineros para el desarrollo del resto de los espacios productivos (Sariego Rodríguez, 1992), más la falta de cohesión entre los braceros, la coexistencia entre distintas formas de trabajo libre y la utilización de variados mecanismos -por parte de los mineros y de las autoridades coloniales-, para disponer de trabajadores forzados (Povea Moreno, 2013). Todos estos supuestos fueron trabajados en relación a las fuentes, contabilizando y haciendo porcentajes sobre las prácticas locales, clasificando a las mismas, citando ejemplos y desarrollándolos (Pelozatto Reilly, 2017c: 13-20), para arribar a conclusiones como que la minería funcionó como principal sector productor y exportador, y como incentivo fundamental para las especializaciones periféricas, que hubo una vinculación entre los yacimientos de plata y las haciendas ${ }^{9}$ de diversos

las autoridades citadinas, con el principal objetivo de controlar las existencias, de que se respetara la delimitación de los espacios productivos urbanos y rurales, y de tener un acceso más fácil a aquellos animales que presentaban múltiples posibilidades de utilización.

9 En territorio novohispano, la utilización de la palabra hacienda hacía referencia más que nada a grandes unidades productivas de un perfil productivo variable, según el lugar y el contexto histórico que se consideren. 
tipos (de beneficio, mixtas ${ }^{10}$, agrícolas y ganaderas), que las intromisiones concejiles fueron diversas pero concentradas en asuntos mineros, y que también tuvieron lugar discusiones más puntuales, como las que se dieron en torno a la puesta en funcionamiento de la Casa de la Moneda (Pelozatto Reilly, 2017c: 20-21).

Gracias a avances como los sintetizados en este apartado, podemos afirmar que el cabildo hispano-colonial (aún durante la misma época), contaba con atribuciones que cambiaban según la región, la geografía del lugar, las labores productivas más consolidadas, los espacios económicos que se fueron configurando en torno a las mismas, los grupos sociales relacionados, el acceso a los medios de producción y los mercados más relevantes, entre otros factores influyentes sobre los cuales las resoluciones del ayuntamiento gozaron de un considerable protagonismo.

\section{Una misma palabra, distintos significados: los hacendados en el Río de la Plata durante el siglo XVIII}

Por último, me gustaría hacer mención y señalar algunos aspectos en torno a una cuestión conceptual: sobre la utilización del término hacendados por parte de las autoridades y de los actores sociales en el Litoral Rioplatense durante distintos momentos en el siglo XVIII. A lo largo de mis investigaciones precedentes, varias de ellas presentadas en eventos universitarios, me encontré con que no era lo mismo lo que se entendía como tales en Santa Fe que en Buenos Aires y, dentro de ésta última, no se empleaba el concepto de la misma manera durante la primera mitad de la centuria en cuestión o hacia finales de la misma.

Gracias al análisis descriptivo y sistemático de las ya mencionadas fuentes municipales de cada ciudad, padrones rurales, registros oficiales de vecinos criadores, sucesiones, inventarios y tasaciones de bienes de productores agropecuarios, podría establecer algunas precauciones que considero necesarias a la hora de estudiar a los ocupantes de las distintas unidades productivas en el Río de la Plata colonial:

1) En todos los casos, se hacía referencia a dueños de haciendas o a pobladores con acceso a la explotación económica del ganado, variando según el caso, el lugar y el contexto histórico (Pelozatto Reilly, 2017a y 2017e).

10 Extensiones rurales de producción agrícola y ganadera diversificada, es decir, orientadas hacia varias demandas mercantiles al mismo tiempo. 
2) Varias veces pude encontrarme con vecinos denominados bajo ese término haciéndose cargo del abasto de carne local, tanto en Buenos Aires como en Santa Fe, a través de todos los mecanismos ya señalados y explicados para el bastimento municipal (Pelozatto Reilly, 2014a y 2017e).

3) Tras haber extraído datos de otras fuentes de carácter más demográfico, como los padrones de Buenos Aires (1726, 1738 y 1744), se puede apreciar que los mismos eran, por lo general, pequeños y medianos productores, poniendo en duda la antigua visión de que en la campaña bonaerense colonial habían predominado los grandes terratenientes ganaderos (Azcuy Ameghino, 1995).

4) Tomando fuentes de los últimos años del siglo XVIII, como el famoso Padrón de Hacendados de Cañada de la Cruz (1789), nos hallamos con que dentro del mismo fueron incluidos no solamente los dueños de mayores extensiones de tierras y de los planteles de ganado más numerosos, sino la gran mayoría de modestos pastores, que no poseían -en un considerable porcentaje de los casos-, más que unas pocas cabezas de ganado (Pelozatto Reilly, 2014b).

5) Vale la pena remarcar que la mayoría de los pequeños y medianos pastores y labradores no eran propietarios de las tierras donde se encontraban asentados y trabajando (Garavaglia, 1993 y 1999), y que accedían a las mismas por medio de distintos vínculos basados en la fuerza de la costumbre, como el peonaje, la agregación y el arrendamiento (Fradkin, 1995; Mayo, 2004).

6) Como rasgo común, al menos para toda la centuria en Buenos Aires, se podría afirmar la creciente importancia de la ganadería vacuna y sus mercados, y de un perfil más bien diversificado y mixto dentro de las unidades productivas rurales (tanto en las más grandes como en las más pequeñas), en cuanto existía una notoria complementariedad entre las distintas especies de ganado y la agricultura (fundamentalmente la de los cereales) (Pelozatto Reilly, 2014a y 2017e), a diferencias de otros períodos en los que tuvieron más peso los caballos y las vacas de manera casi excluyente (Pelozatto Reilly, 2017b).

7) Si bien la terminología era aplicada para productores "laicos", no debemos pasar por alto la importancia de la Iglesia católica y sus representantes como actores económicos activos, involucrados en las distintas prácticas pecuarias (vaquerías, recogidas de ganado alzado, cría de domésticos) y a la propiedad de buenas cantidades de riquezas monetarias, en bienes inmuebles, esclavos, etc. (Fradkin, 2000). Tal fue el caso, por ejemplo, de los jesuitas, quienes tuvieron famosas diferencias con los municipios de Santa Fe y Buenos Aires en torno a la apropiación de los bovinos cimarrones disponibles en los campos de la actual Provincia de Entre Ríos y de la Banda Oriental del Uruguay (Pelozatto Reilly, 2017a). 
8) Considerando los resultados de todas mis investigaciones citadas, puedo afirmar que, a través de las resoluciones capitulares en relación a las participaciones de los hacendados, es posible divisar varios puntos de interés, como los mercados a los cuales se encontraban vinculados, las características de sus estancias y rodeos, su participación en el marco de las diferentes labores agropecuarias, el grado de representación que tenían dentro de la corporación municipal, etc.

\section{Algunas consideraciones parciales}

Luego de repasar toda una serie de trabajos que he venido realizando en el último tiempo, me parece oportuno enumerar algunas conclusiones generales acerca del cabildo hispanoamericano, el carácter de sus políticas económicas y su influencia sobre los distintos mercados, teniendo en cuenta las diferencias locales, regionales y cronológicas:

1) Las intervenciones sobre cuestiones de índole económica (producción, comercio, abastos, etc.) variaron según el municipio y la orientación productiva que tuviese (por ejemplo: la ganadería y sus variantes en el Río de la Plata, la importancia del bastimento local en diferentes puntos del actual Ecuador, la minería de la plata y sus problemas en Zacatecas, entre otros).

2) Dentro de mis obras hay una clara concentración en la región conocida como Litoral Rioplatense (fundamentalmente dentro de los dominios de los cabildos de Buenos Aires y Santa Fe), por obvias razones (mayor disponibilidad de fuentes documentales y bibliográficas), a través de las cuales pude abordar diversos asuntos de interés dentro de la actividad agropecuaria y sus variantes, según el periodo, el objeto de estudio, y los planteos teórico-metodológicos: abasto de carne local, producción y exportación de cueros (Pelozatto Reilly, 2014a; 2017a y 2017b), la configuración de los espacios productivos y sus orientaciones (Pelozatto Reilly, 2017b), las similitudes y diferencias existentes entre distintas ciudades (Pelozatto Reilly, 2017e), la evolución de determinadas alternativas ganaderas y de los productores rurales a lo largo del tiempo (Pelozatto Reilly, 2014b y 2017b), los conflictos que tuvieron lugar por el usufructo de los recursos económicos más relevantes (Pelozatto Reilly, 2017b), etc.

3) A lo largo de este escrito logré sostener las hipótesis planteadas al principio, exponiendo, por separado, distintos tipos de indagaciones históricas (contraste entre espacios locales, regiones y periodos, tratamiento de una misma problemática en dos o más municipios, y algunas discusiones de tipo más bien conceptual). 
Para finalizar, me agradaría mencionar algunos abordajes -tanto generales como específicos- que considero pendientes dentro de mi área de especialización, y posibles líneas de investigación:

- La participación de los productores agropecuarios en los mercados regionales y exteriores.

- Su relación con los principales puestos de la administración pública.

- Sus condiciones de vida, relaciones sociales, parentales, alianzas políticas, etc.

- Sus vínculos con el comercio al por menor y en grandes cantidades.

- Los tipos de mano de obra que utilizaban en sus prácticas y establecimientos.

- En lo que respecta a la ganadería rioplatense en particular, la evolución de los mercados y de los precios pecuarios, sus causas, consecuencias y resoluciones locales al respecto.

- Avanzar sobre todos estos temas en otros sitios del actual territorio argentino, puesto que alcanzan las fuentes como para encarar más indagaciones de historia local.

Respecto de los avances que pude hacer para los casos ecuatorianos, me gustaría:

- En lo tocante a distritos mineros, como la Ciudad de Zacatecas, destacar los nexos existentes entre los miembros de la elite local, la minería y otro tipo de labores y unidades productivas.

- Siguiendo lo planteado en el ítem anterior, profundizar sobre la relación entre la extracción platera y el desarrollo de las haciendas de diverso tipo, mientras que también podría hacerse algo desde el punto de vista de lo social.

- Dar continuidad a los estudios comparativos, tomando localidades de una misma región o incluso espacios muy dispares, durante el mismo periodo temporal, y hasta pensar en la posibilidad de contrastar recortes cronológicos para un mismo lugar.

- Complementar el análisis de una variedad más amplia de documentos y de fuentes bibliográficas, según las necesidades propuestas por cada objeto de estudio. 


\title{
Referencias bibliográficas
}

\author{
a) Artículos y capítulos de libros
}

Commons, A. (1989). "Principales zonas mineras en la segunda mitad del siglo XVIII", en Revista Investigaciones Geográficas, № 20, pp. 105-120.

Fradkin, R. (1995). "Según la costumbre del pays: costumbre y arriendo en Buenos Aires durante el siglo XVIII", en Boletín del Instituto de Historia Argentina y Americana "Dr. E. Ravignani", Tercera Serie, No 1, pp. 39-64.

Fradkin, R. (2000). "El mundo rural colonial", en Tandeter, E. (Dir.) Nueva Historia Argentina. Tomo II. La sociedad colonial. Buenos Aires: Editorial Sudamericana, pp. 241-284.

Garavaglia, J. C. (1993). "Las estancias en la campaña de Buenos Aires. Los medios de producción (1750-1850)", en Fradkin, R. (Comp.). La historia agraria del Río de la Plata colonial. Los establecimientos productivos (II). Buenos Aires: Centro Editor de América Latina, pp. 124-208.

Gil Montero, R. (2012). "Tecnología minera en los siglos XVI-XIX: una perspectiva comparada", en Bodemer, K. (Coord.). Cultura, sociedad y democracia en América Latina. Aportes para un debate multidisciplinario. España: Biblioteca Iberoamericana, pp. 306-326.

González Lebrero, R. (1993). "Chacras y estancias en Buenos Aires a principios del siglo XVII", en Fradkin, R. (Comp.). La historia agraria del Río de la Plata colonial. Los establecimientos productivos (II). Buenos Aires: Centro Editor de América Latina, pp. 70-123.

Moncada González, G. (2009). "Políticas de abasto de alimentos en la ciudad de México en los albores de la guerra de Independencia 1810-1812", en Long Towel, J. y A. Attolini Lecón (Coord.). Caminos y mercados de México. México: Instituto de Investigaciones Históricas de la UNAM, Instituto Nacional de Antropología e Historia.

Pelozatto Reilly, M. L. (2014a). "El Cabildo, la ganadería y el abasto local en el litoral rioplatense, 1723-1750", en Actas de las Quintas Jornadas de Historia Regional de La Matanza, pp. 230-244.

Pelozatto Reilly, M. L. (2014b). "La estancia rioplatense a fines del siglo XVIII: Ios "hacendados" de Cañada de la Cruz (1789)", en II Jornadas de Investigación "Rogelio C. Paredes", Universidad de Morón, 14-XI-2014.

Pelozatto Reilly, M. L. (2015). "El Cabildo, los vecinos y la utilización de la "otra banda" como territorio alternativo en la economía rural colonial. Buenos Aires y Santa Fe durante la extinción del ganado cimarrón y las vaquerías tradicionales (1720-1750)", en Revista Estudios Históricos, Vol. VII, No 14, Uruguay. 
Pelozatto Reilly, M. L. (2016). "Ganadería y unidades productivas mixtas en Buenos Aires colonial, 1726-1759", en Revista de Historia y Geografía, № 34, Universidad Católica Silva Henríquez, Chile, pp. 43-65.

Pelozatto Reilly, M. L. (2017a). "Reducciones y vaquerías en el Río de la Plata Colonial. Intervenciones capitulares y negociaciones con la Compañía de Jesús en torno a la explotación del ganado vacuno cimarrón. Santa Fe y Buenos Aires, 16771726", en Jornadas Estudios sobre religión, sociedad y cultura, UCA, 13-VI-2017.

Pelozatto Reilly, M. L. (2017b). "Mercedes de tierras, repartimientos de indios y conformación de las primeras unidades productivas rurales en Buenos Aires colonial (1580-1590)", en I Encuentro de Historia de Barracas al Norte y al Sud, Asociación Fraga, 26-VIII-2017.

Pelozatto Reilly, M. L. (2017c). "El cabildo secular y sus diversas intervenciones en torno a la minería de la plata en Nueva España colonial: Zacatecas (Nueva Galicia), 1581-1603", en IV Jornadas de Estudiantes y Graduados de Historia, Universidad Nacional de General Sarmiento, 19-IX-2017.

Pelozatto Reilly, M. L. (2017d). "Funciones del cabildo, prácticas económicas y mercados en el Ecuador colonial (Quito y Guayaquil, segunda mitad del siglo $X V I)$ ", en XI Jornadas de Historia Argentina y Latinoamericana, IES No 1 "Alicia Moreau de Justo", 12-X-2017.

Pelozatto Reilly, M. L. (2017e). "Ganadería, intervenciones capitulares y mercados durante la primera mitad del siglo XVIII. Análisis y comparación de distintas jurisdicciones municipales: Santa Fe y San Luis", en XII Jornadas de Estudios Sociales de la Economía, IDAES, UNSAM, 14-XI-2017.

Povea Moreno, I. (2013). "Mano de obra libre en la minería del mercurio y la plata, siglo XVIII: el caso de Huancavelica, Potosí y Zacatecas", en Cava Mesa, B. (Coord.). América en la memoria: Conmemoraciones y reencuentros. Tomo II, Asociación Española de Americanistas, pp. 383-396.

Quiroz, E. (2011). "Entre el humanismo y el mercantilismo. El bien común en el abasto de carne de la ciudad de México, 1708-1716", en Cuadernos de Historia, Universidad de Chile, № 35, pp. 35-59.

Sariego Rodríguez, J.L. (2012). "Minería y territorio en México: tres modelos históricos de implantación socioespacial", en Ería: Revista cuatrimestral de Geografía, № 29, pp. 197-210.

\section{b) Libros}

Azcuy Ameghino, E. (1995). El latifundio y la gran propiedad colonial rioplatense. Buenos Aires: Fernando García Cambeiro. 
Coni, E. (2011). Historia de las vaquerías de Río de la Plata (1555-1750). Alicante: Biblioteca Virtual Miguel de Cervantes.

Fradkin, R. y J. C. Garavaglia (2009). La Argentina colonial. El Río de la Plata entre los siglos XVI y XIX. Buenos Aires: Siglo XXI Editores.

Garavaglia, J. C. (1999). Pastores y labradores de Buenos Aires. Una historia agraria de la campaña bonaerense 1700-1830. Buenos Aires: Ediciones de la flor.

Halperín Donghi, T. (2010). Historia contemporánea de América Latina. Buenos Aires: Alianza Editorial.

Long Towel, J. y A. Attolini Lecón (Coord.) (2009). Caminos y mercados de México. México: Instituto de Investigaciones Históricas de la UNAM, Instituto Nacional de Antropología e Historia.

Mayo, C. (2004). Estancia y sociedad en la pampa (1740-1820). Buenos Aires: Editorial Biblos. 